\title{
Predicting Outcomes Using the Heart Failure Survival Score in Adults with Moderate or Complex Congenital Heart Disease
}

\author{
Elaine Y. Lin, MD*, Hillel W. Cohen, $\mathrm{DrPH}^{\dagger}$, Ami B. Bhatt, MD ${ }^{\ddagger}$, Ada Stefanescu, MDCM ${ }^{\ddagger}$, \\ David Dudzinski, MD JD ${ }^{\ddagger}$, Doreen DeFaria Yeh, MD ${ }^{\ddagger}$, Jacob Johnson, MD $\ddagger$, and George K. \\ Lui, MD ${ }^{\star}, \S$ \\ "Division of Cardiology, Department of Medicine, Montefiore Medical Center, Albert Einstein \\ College of Medicine, Bronx, NY \\ †Department of Epidemiology \& Population Health, Albert Einstein College of Medicine, Bronx, \\ NY \\ ¥Division of Cardiology, Institute for Heart, Vascular, and Stroke Care, Massachusetts General \\ Hospital, Harvard Medical School, Boston, MA \\ $\S$ Division of Cardiovascular Medicine, Department of Medicine Stanford University School of \\ Medicine, Stanford, CA
}

\section{Abstract}

Background-Adults with congenital heart disease (CHD) face increased risk for morbidity and mortality with age, but few prognostic models exist.

Objective-This study aims to assess whether the Heart Failure Survival Score (HFSS), which risk stratifies patients for heart transplantation, predicts outcomes in adults with moderate or complex CHD.

Methods-This was a multicenter, retrospective study which identified 441 patients with moderate or complex CHD between 2005 and 2013, of whom 169 had all the HFSS parameters required to calculate the risk score. Because all study patients were deemed low risk by the HFSS, the score was dichotomized at the median (10.4). Outcomes included death, transplant or ventricular assist device (VAD), arrhythmia requiring treatment, non-elective cardiovascular (CV) hospitalizations, and the composite. Associations of mean HFSS and HFSS $<10.4$ with each outcome were assessed.

Results-The cohort had mean $\pm \mathrm{SD}$ age of $33.6 \pm 12.6$ years, peak $\mathrm{VO}_{2} 21.8 \pm 7.5 \mathrm{~mL} / \mathrm{kg} / \mathrm{min}$, HFSS of $10.45 \pm 0.88$, and median years follow-up of $2.7(1.1,5.2)$. There were 5 deaths $(2.8 \%)$, no transplants or VADs, 25 arrhythmias (14.8\%), $22 \mathrm{CV}$ hospitalizations (13\%), and 39 composites $(23.1 \%)$. Lower mean HFSS was observed for patients who died (9.6 \pm 0.83 vs. $10.5 \pm 0.87, \mathrm{p}=$ $0.02)$, arrhythmia requiring treatment ( $10.0 \pm 0.70$ vs. $10.5 \pm 0.89, \mathrm{p}=0.005), \mathrm{CV}$ hospitalizations $(9.9 \pm 0.73$ vs. $10.5 \pm 0.88, \mathrm{p}=0.002)$, and the composite $(10.0 \pm 0.70$ vs. $10.6 \pm 0.89, \mathrm{p}<0.001)$.

Address for Correspondence: Elaine Y. Lin, New York Hospital Queens, 56-45 Main Street, WA\#200, Flushing, NY 11355, Tel: (646)784-2737 Fax: (917)210-3965, tingtinglin@gmail.com.

Disclosure: There is no relationship with industry pertaining to this manuscript. 
The positive and negative predictive values of HFSS < 10.4 for the composite were $34 \%$ and $88 \%$ respectively, with sensitivity and specificity $74 \%$ and $56 \%$.

Conclusions-Although a low HFSS was significantly associated with outcomes, it did not adequately risk stratify adults with CHD, whose heterogeneous pathophysiology differs from that of the acquired heart failure population. Further studies are warranted to provide a more accurate prognosis.

\section{Keywords}

prognosis; ACHD; mortality; morbidity; risk; model

\section{Introduction}

Congenital heart disease (CHD) composes nearly one-third of all the congenital anomalies, posing a substantial challenge to healthcare systems globally (1). With a growing CHD population whose life expectancy is growing in parallel, we are now faced with new clinical questions regarding long-term morbidity and mortality in these patients. Many studies have identified important, independent predictors of mortality in adults with CHD. However, few studies exist which attempt to establish a risk score $(2,3)$. Given the paucity of widely applicable prognostic model for adults with moderate to complex CHD, this study aims to evaluate an existing model designed for the general heart failure population to risk stratify CHD patients. The Heart Failure Survival Score (HFSS) was derived and validated by Aaronson et al to risk stratify ambulatory patients with chronic, advanced heart failure for possible cardiac transplantation (4). We sought to determine whether the HFSS could predict outcomes in adults with moderate or complex CHD lesions.

\section{Methods}

\section{Study Patients}

This was a multicenter, retrospective observational study conducted by Montefiore Medical Center in Bronx, NY, and Massachusetts General Hospital in Boston, MA. We included all adults $\geq 18$ years old, with moderate or complex congenital heart disease $(5,6)$ within the study period (June 2005 to February 2013), who had completed cardiopulmonary exercise testing (CPX) within 1 year of the initial visit with a cardiologist or congenital heart disease specialist. Cases were identified via ICD-9 codes by using institution-specific databases, paper charts, and electronic medical records. Patients who did not have all the parameters required to calculate the HFSS, including various laboratory values, were excluded.

Baseline data were collected at the initial visit with the cardiologist in the adult congenital heart clinics. The congenital heart lesions considered hemodynamically most important were recorded as the main diagnosis, and severity of the CHD diagnosis was assigned according to the Besthesda classification (7). Additional measures included age, height, weight, race, ethnicity, number of cardiovascular surgeries, New York Heart Association (NYHA) class, systemic ejection fraction (EF), presence of cyanosis, labs including serum sodium, hemoglobin, and creatinine, device type, cardiopulmonary exercise testing (CPX) parameters, and medications. The systemic ventricular ejection fraction (EF) was obtained 
from echocardiographic studies within one year of the initial visit. However, if the patient had a cardiac magnetic resonance imaging, this systemic EF was used instead.

Cyanosis was defined as an oxygen saturation of less than $90 \%$ at rest. Heart rate reserve was defined as peak heart rate minus resting heart rate. Chronotropic incompetence was defined as the inability to reach $80 \%$ of the heart rate reserve.

CPX was performed according to the protocols at each center. Peak oxygen consumption (peak $\mathrm{VO}_{2} ; \mathrm{mL} / \mathrm{kg} / \mathrm{min}$ ) was assessed during ramp cycle ergometer or graded treadmill exercise (Naughton, modified Bruce, or Bruce protocol). Patients exercised to a symptomlimited maximum. Heart rate, blood pressure, oxygen saturation, and ECG were measured continuously. Expired gases were collected throughout the protocol, and oxygen consumption was calculated on a breath-by-breath basis. Peak $\mathrm{VO}_{2}$ was defined as the highest value of oxygen uptake attained in the final 20 seconds of exercise when the respiratory exchange ratio (RER) was $\geq 1.05$. At Massachusetts General Hospital, peak $\mathrm{VO}_{2}$ was defined as the highest oxygen uptake measured during the last minute of symptomlimited exercise.

The study was approved by the institutional review boards at each institution.

\section{Outcomes and Follow-up}

All patients were followed up in the adult CHD clinic between June 2005 to February 2013. Outcomes included death, cardiac transplant or VAD, arrhythmias requiring treatment, $\mathrm{CV}$ admissions, and a composite defined as the presence of any of those outcomes. If a patient has an arrhythmia requiring hospitalization, the recorded outcomes would include arrhythmias requiring treatment, $\mathrm{CV}$ hospitalization, and the composite. Arrhythmias requiring treatment are defined as any non-elective (including outpatient) treatment of sustained arrhythmias. CV admissions are defined as any non-elective cardiovascular hospitalizations over 24 hours.

Follow-up is defined as time from the first documented visit with general cardiology or adult CHD specialist to the first specified event or study end. At Montefiore Medical Center, all outcomes including death were assessed via institution-specific databases with links to the social security death tapes enabling identification of deaths outside the institutions. At the Massachusetts General Hospital, outcomes including death were also assessed via institution-specific databases, but there were no links to the social security death tapes. Deaths and admissions outside the institution were based on physicians receiving report from other health care providers.

\section{HFSS and Risk Stratification}

The HFSS was calculated based on previously published and prospectively validated algorithms, and patients were assigned to a risk stratum according to the score (4). The HFSS combines several known independent predictors of mortality in the general heart failure population, and it is derived as the sum of the absolute values of coefficients assigned to each variable. Components of the HFSS include presence or absence of ischemia, presence or absence of interventricular conduction delay (defined as QRS duration greater 
than or equal to 120 milliseconds), resting heart rate, left ventricular ejection fraction (LVEF), mean blood pressure, serum sodium, and peak $\mathrm{VO}_{2}$. Due to the anatomic variations in our study cohort, the systemic, and not the left ventricular ejection fraction, was used in the calculation of HFSS. The score is computed based on the published algorithms, and the conventional risk strata are defined as low (HFSS >8.10), medium (7.2-8.09), and high ( 3.19 ). Low risk confers a 1-year survival of $88 \%$, while high risk corresponds to a 1-year survival of $35 \%$, free of urgent cardiac transplantation.

\section{Statistical Analysis}

Characteristics were summarized by mean \pm sd or median (interquartile range) for continuous measurements and by percentages for categorical measurements and compared across groups with independent samples $t$-test, Mann Whitney $\mathrm{U}$ test or chi-square as appropriate. HFSS was assessed as a continuous variable and also dichotomized at the median 10.4. The distribution of primary type of CHD and outcomes were examined by percentages. The sensitivity, specificity, and positive and negative predictive values were calculated for HFSS $<10.4$ predicting the composite outcome. Logistic regression models were constructed for the composite outcome using dichotomized HFSS and adding covariates with significant bivariate associations with the outcome. Logistic models were assessed for goodness of fit with a Hosmer-Lemeshow test. A two-tailed alpha of 0.05 was used to denote statistical significance and analyses were performed with SPSS for windows software (version 20).

The authors had full access to and take full responsibility for the integrity of the data. All authors have read and agree to the manuscript as written.

\section{Results}

A total of 441 adult patients with moderate or complex congenital heart disease were identified. Two hundred sixty-one patients were excluded due to the lack of CPX results. After excluding 11 patients with other missing parameters required by the HFSS, 169 were available for analysis (Fig. 1).

\section{Baseline Characteristics}

The majority of patients carried the diagnosis of repaired tetralogy of Fallot ( $\mathrm{n}=73,43.2 \%)$, with a relatively even distribution of other lesions including Ebstein anomaly, systemic right ventricle, and single ventricle with Fontan circulation (Fig. 2). Fifteen patients (9\%) had cyanosis.

Fifty three percent were female and most were in NYHA Class I and II (Table 1). The mean age was $33.6 \pm 12.6$ years. Most of the patients did not have a history of CV hospitalization, arrhythmia, or device implantation. Thirty-six percent of the patients were taking beta blockers, $24.1 \%$ were taking angiotensin converting enzyme inhibitors, and $7.1 \%$ angiotensin II receptor blockers.

Mean \pm sd peak $\mathrm{VO}_{2}$ was $21.8 \pm 7.5 \mathrm{~mL} / \mathrm{kg} / \mathrm{min}$ for all patients in the cohort. $89.3 \%$ of the patients reached a respiratory exchange ratio over 1 . The mean $\pm \mathrm{sd} \mathrm{VE} / \mathrm{VCO}_{2}$ slope was 33.2 \pm 7.8 . By lesion category, patients with complex cyanotic lesions had the lowest mean peak 
$\mathrm{VO}_{2}$ of $17.2 \pm 5.6 \mathrm{~mL} / \mathrm{kg} / \mathrm{min}$, and those with atrioventricular septal defect had the highest mean peak $\mathrm{VO}_{2}$ of $25.8 \pm 8.5 \mathrm{~mL} / \mathrm{kg} / \mathrm{min}$.

Thirteen (7.7\%) of the patients had oxygen saturations $\geq 90 \%$ at rest but had desaturations to $<90 \%$ during exercise. Fifteen $(8.9 \%)$ of the patients had oxygen saturations $<90 \%$ and had further desaturations during exercise.

The mean heart rate reserve was $65 \pm 28$ beats per minute. The average predicted peak heart rate was $74 \%$. One patient had chronotropic incompetence, was on a beta blocker but did not have a pacemaker.

The variables associated with the composite event are shown in Table 2.

\section{HFSS and Outcomes}

During a median (interquartile range) follow-up of $2.7(1.1,5.2)$ years, 5 patients $(2.8 \%)$ died, all of acute decompensated heart failure. There were no cardiac transplantation or VADs. Twenty-two (13\%) patients had at least one non-elective CV hospitalization over 24 hours, 25 (14.8\%) patients had at least one arrhythmia requiring treatment (Fig. 3), and 39 patients had at least one of these outcomes (composite).

The mean HFSS in the study cohort was $10.45 \pm 0.89$. Table 3 shows the average values of the individual components used to derive the HFSS. Notably, coronary artery disease was absent in all patients, and more than half had interventricular conduction delay with QRS duration over 120 milliseconds.

Although all of the study patients had HFSS $>8.1$, the conventional threshold for low risk, there was a significant difference in peak $\mathrm{VO}_{2}$ for patients with HFSS $<10.4$ compared to those with HFSS $\geq 10.4(19.8 \pm 6.8$ vs. $23.9 \pm 7.7, \mathrm{p}<0.001)$. For patients with HFSS $<10.4$, the odds ratios for arrhythmia, CV hospitalization, and the composite were 3.6 (95\% CI 1.4-9.6, $\mathrm{p}=0.007), 3.8(95 \%$ CI 1.3-11.0, $\mathrm{p}=0.008)$, and 3.7 (95\% CI 1.7-8.2, $\mathrm{p}=$ 0.001 ), respectively (Table 4). The positive and negative predictive values of HFSS $<10.4$ for the composite were $34 \%$ and $88 \%$ respectively, with sensitivity and specificity $74 \%$ and $56 \%$.

A lower mean HFSS was observed for those with versus those without outcome: death (9.6 \pm 0.83 vs. $10.5 \pm 0.87, \mathrm{p}=0.02)$, arrhythmias $(10.0 \pm 0.70$ vs. $10.5 \pm 0.89, \mathrm{p}=0.005), \mathrm{CV}$ hospitalizations $(9.9 \pm 0.73$ vs. $10.5 \pm 0.88, \mathrm{p}=0.002)$, and composite $(10.0 \pm 0.70$ vs. 10.6 $\pm 0.89, \mathrm{p}<0.001)$. Figure 4 illustrates the distribution of HFSS in patients with and without the composite outcome.

A binary logistic regression model using a forward stepwise procedure was constructed for the composite outcome. All variables significantly associated with HFSS $<10.4$ were available to enter the model along with HFSS (Table 2). Only systemic EF, history of CV hospitalization, use of spironolactone and NYHA class greater than II remained in the model. The final model had a Nagelkerke $\mathrm{R}^{2}$ of $0.28(\mathrm{p}<0.001)$ and was able to predict $95 \%$ of the non-events but only $38 \%$ of the positive composite events. 


\section{Discussion}

The population of adults with congenital heart disease continues to age with increasing morbidity and mortality. Deaths and hospitalizations in CHD have shifted from infancy to adulthood $(8,9)$. Prior studies have focused on parameters of cardiopulmonary exercise test or arrhythmia as a predictor of survival in adults with congenital heart disease $(2,3,10-12)$, This study demonstrated that a well-accepted risk score for the general heart failure population, HFSS, did not accurately risk stratify adults with congenital heart disease for survival or other outcomes such as hospitalization and arrhythmia.

A lower HFSS was overall significantly associated with worse outcomes even though all of our patients were classified as low risk by conventional thresholds. Events including death, CV hospitalization, and arrhythmias requiring treatment occurred in these patients deemed low risk. It is plausible that the HFSS was found to have a poor positive predictive value in patients with moderate to complex CHD as it did include the key risk factors of this population. For instance, although most CHD had interventricular conduction delay, it is mostly due to prior surgeries rather than the underlying cardiomyopathy. As shown in Table 5, most ACHD patients have right bundle branch block on the electrocardiogram. In contrast, the QRS morphology of most acquired heart failure patients is often left bundle branch block, which is indicative of a diseased myocardium and is an independent predictor of mortality in this population (13). Furthermore, none of the CHD patients had coronary artery disease or ischemia during extensive chart review, one of the key factors in HFSS algorithms. However, this may be underrepresented because there was no formal evaluation of coronary artery disease. The average systemic ventricular EF in this cohort was $56 \%$ $\pm 10 \%$. In a general heart failure (HF) population referred for transplantation, the average left ventricular EF is lower at approximately $22 \%$ (4). The key differences between the ambulatory advanced HF patients from the validation cohort of the original HFSS study and ACHD patients in this study are highlighted in Table 6. The ACHD group is younger, has a lower mean NYHA class, higher peak $\mathrm{VO}_{2}$, and higher systemic ventricular $\mathrm{EF}$. The $\mathrm{EF}$ is considered to be that of the left ventricle in HFSS. In adults with congenital heart disease, there is significant heterogeneity in this population's anatomy resulting in right or left morphologic ventricles as the systemic ventricle. The right ventricle (RV) has become the focus of intensive research as the ACHD population grows. RV failure is often the result of persistent abnormalities in intracardiac pressure, volume, and flow due to either the original congenital lesion or as results of corrective surgeries (14). Although the initial "insult" may be drastically different between heart failure due to acquired and congenital etiologies, the mechanism of downstream neurohormonal activation that leads to eventual decompensation may be similar.

In this study, several variables were significantly associated with the composite outcome, including a lower HFSS, lower heart rate reserve, lower peak $\mathrm{VO}_{2}$, lower systemic EF, presence of device, history of arrhythmias and CV hospitalization, the use of spironolactone and diuretic, and NYHA class greater than II (Table 2). Median HFSS alone had a positive predictive value of $34 \%$ and negative predictive value of $88 \%$. In multivariate analysis, the addition of history of CV hospitalization, the use of aldosterone, or both to the HFSS led to 
better accuracy especially with respect to negative predictive value (95\% NPV) but still had a positive predictive value too low to be clinically useful (38\% PPV).

As mentioned previously, the final pathway for disease severity may be common in both congenital and acquired heart failure populations. The aforementioned parameters such as the use of diuretics and spironolactone, as well as the presence of device, are likely markers of disease. Conversely, the absence of these markers portends a favorable prognosis.

The Seattle Heart Failure Model (SHFM) is another prognostic tool frequently used in patients with chronic, advanced heart failure to predict survival (15). It includes multiple easily obtainable variables, such as demographics, laboratory values, vital signs, presence or absence of ischemia or device, and medications with dosages. The SHFM was able to discriminate among adult CHD patients who have a peak $\mathrm{VO}_{2}$ greater or less than $20 \mathrm{~mL} / \mathrm{kg} /$ min, allowing the use of clinically obtained data to infer poor peak $\mathrm{VO}_{2}$ without requiring CPX (16). Furthermore, it was demonstrated that SHFM successfully risk stratifies ACHD patients on several outcomes including mortality and CV hospitalizations. The SHFM was more successful at risk stratifying ACHD patients than the HFSS probably because it is a model that takes into account severity of illness through several clinical and laboratory values. The fact that SHFM, designed for patients with acquired heart failure, was able to separate out high versus low-risk ACHD patients, again supports the concept that the heart failure syndrome, regardless of the systemic ventricular morphology, leads to a common final pathophysiologic pathway. This final common pathway is represented in SHFM because it includes variables such as number of heart failure medications and dose, NYHA class, and several laboratory values not represented in HFSS.

Nevertheless, an accurate prognosis in ACHD remains challenging due to the heterogeneity of this population. In patients with tetralogy of Fallot status-post repair, QRS over 180 milliseconds was the most sensitive predictor of life-threatening ventricular arrhythmias and correlated with chronic RV volume overload (17). In patients with single ventricle physiology, the presence of protein-losing enteropathy, single morphologically right ventricle, high right atrial pressure, and the lack of antiplatelet or anticoagulation therapy have been shown to be independent predictors of death (18). Several individual variables have also been found in prior studies to be significant predictors of mortality in a cohort of adults with CHD including hyponatremia, neurohormonal activation, renal insufficiency, anemia, lower peak $\mathrm{VO}_{2}$, increased $\mathrm{VE} / \mathrm{VCO}_{2}$ slope, and abnormal heart rate reserve (1012, 19-22).

The fact that nearly two-thirds of the study group did not have a CPX and therefore excluded highlights the heterogeneity of the ACHD population and variations in practice patterns. Thus, the development of a risk score or model can be helpful in a number of ways. Although it does not replace the clinician's judgment, it can be used to objectively risk stratify patients in a standardized way. In addition, since there are limited adult CHD centers worldwide, many patients encounter internists, general cardiologists, and other practitioners at symptom onset. A risk score is an easy way for these clinicians to recognize those at high risk and facilitate early referral to a CHD specialist for further management. Even among 
CHD specialists, a prognostic model may be helpful in monitoring effects of medications and other therapies.

Furthermore, such a risk model could help educate patients by providing additional insights into their illness and possibly enhance compliance. Most importantly, a novel risk model for CHD patients could help identify those who require close follow-up, invasive therapies, or referral for cardiac transplantation.

\section{Limitations}

This study had several limitations, including its retrospective nature and the small number of deaths during the study period. The sample size was small, with a relatively short follow-up period. In addition, about $60 \%$ of patients who did not have CPX were excluded. These patients may potentially alter the outcomes of this study were they included in the cohort.

The retrospective design of this study did not allow for a standard analysis of ventricular systolic function among the institutions. The ejection fraction is mostly qualitative and may be subjective based on the interpreting echocardiographer except for cases where cardiac magnetic resonance (CMR) imaging techniques were used. However, the subaortic ventricular systolic function was reported by experienced echocardiographic readers at the adult congenital cardiac centers. Future study should include a quantitative analysis of ejection fraction by CMR to further evaluate the risk of systemic ventricular dysfunction and outcome.

Finally, CV hospitalizations or arrhythmias requiring treatment that occurred outside of the study institutions may not have been known and excluded. In addition, the HFSS was extrapolated to predict both mortality and morbidity, but the latter has not been validated. It was also extrapolated to predict outcomes in adult CHD patients whose overall morbidity and mortality rates are lower than the general heart failure population for which HFSS was originally designed.

\section{Conclusions}

Although a low HFSS was significantly associated with outcomes, it failed to adequately risk stratify adult patients with CHD, whose heterogeneous pathophysiology differs from that of the general heart failure population. Further studies are warranted to provide accurate prognosis in adult CHD patients.

\section{Acknowledgement}

This publication was supported in part by the CTSA Grant 1 UL1 TR001073-01, 1 TL1 TR001072-01, 1 KL2 TR001071-01 from the National Center for Advancing Translational Sciences (NCATS), a component of the National Institutes of Health (NIH), Bethesda, MD.

\section{Abbreviations}

$\begin{array}{ll}\text { CHD } & \text { congenital heart disease } \\ \text { VAD } & \text { ventricular assist device }\end{array}$




$\begin{array}{ll}\text { CV } & \text { cardiovascular } \\ \text { HFSS } & \text { Heart Failure Survival Score } \\ \text { CPX } & \text { cardiopulmonary exercise testing } \\ \text { NYHA } & \text { New York Heart Association } \\ \text { EF } & \text { ejection fraction } \\ \text { ACHD } & \text { adult congenital heart disease } \\ \text { SHFM } & \text { Seattle Heart Failure Model }\end{array}$

\section{References}

1. Dolk H, Loane M, Garne E. for the European Surveillance of Congenital Anomalies (EUROCAT) Working Group. Congenital heart defects in Europe: prevalence and perinatal mortality: 2000 to 2005. Circulation. 2011; 123:841-849. [PubMed: 21321151]

2. Yap SC, Harris L, Chauhan VS, Oechslin EN, Silversides CK. Identifying high risk in adults with congenital heart disease and atrial arrhythmias. Am J Cardiol. 2011; 108:723-728. [PubMed: 21684512]

3. Inuzuka R, Diller GP, Borgia F, Benson L, Tay EL, et al. Comprehensive use of cardiopulmonary testing identifies adults with congenital heart disease at increased mortality risk in the medium term. Circulation. 2012; 125:250-259. [PubMed: 22147905]

4. Aaronson KD, Schwartz SJ, Chen TM, Wong KL, Goin JE, et al. Development and prospective validation of a clinical index to predict survival in ambulatory patients referred for cardiac transplant evaluation. Circulation. 1997; 95(12):2660-2667. [PubMed: 9193435]

5. Warnes CA, Williams RG, Bashore TM, Child JS, Connolly HM, et al. ACC/AHA 2008 guidelines for the management of adults with congenital heart disease. JACC. 2008; 52:143-263.

6. Marelli AJ, Mackie AS, Ionescup-Ittu R, Rahme E, Pilote L. Congenital heart disease in the general population: Changing prevalence and age distribution. Circulation. 2007; 115:163-172. [PubMed: 17210844]

7. Webb GD, William RG. $32^{\text {nd }}$ Bethesda Conference:"Care of the Adult with Congenital Heart Disease". JACC. 2001; 37(5):1161-1198.

8. Khairy P, Ionescu-Ittu R, Mackie AS, Abrahamowicz M, Pilote L, et al. Changing mortality in congenital heart disease. JACC. 2010; 56:1149-1157. [PubMed: 20863956]

9. O’Leary JM, Siddhiqi OK, De Ferranti S, Landzberg MJ, Opotowsky AR. The changing demographics of congenital heart disease hospitalizations in the United States: 1998 through 2010. JAMA. 2013; 309(10):984-986. [PubMed: 23471480]

10. Diller GP, Dimopoulos K, Okonko D, Uebing A, Broberg C, et al. Heart rate response during exercise predicts survival in adults with congenital heart disease. JACC. 2006; 48(6):1250-1256. [PubMed: 16979014]

11. Diller GP, Dimopoulos K, Okonko D, Li W, Babu-Narayan S, et al. Exercise intolerance in adult congenital heart disease: comparative severity, correlates, and prognostic implication. Circulation. 2005; 112:828-835. [PubMed: 16061735]

12. Dimopoulos K, Okonko D, Diller GP, Broberg CS, Salukhe TV, et al. Abnormal ventilatory response to exercise in adults with congenital heart disease relates to cyanosis and predicts survival. Circulation. 2006; 113:2796-2802. [PubMed: 16769913]

13. Baldasseroni S, Opasich C, Gorini M, Lucci D, Marchionni N, et al. Left bundle-branch block is associated with increased 1-year sudden and total mortality rate in 5517 outpatients with congestive heart failure: a report from the Italian network on congestive heart failure. Am Heart J. 2002; 143(3):398-405. [PubMed: 11868043] 
14. Bolger AP, Coats AJS, Gatzoulis MA. Congenital heart disease: the original heart failure syndrome. Eur Heart J. 2003; 24:970-976. [PubMed: 12714029]

15. Levy W, Mozaffarian D, Linker D, Sutradhar SC, Anker SD, et al. The Seattle Heart Failure Model: Prediction of survival in heart failure. Circulation. 2006; 113:1424-1433. [PubMed: 16534009]

16. Stefanescu A, Macklin E, Lin E, Dudzinski DM, Johnson J, et al. Usefulness of the Seattle Heart Failure Model to identify adults with congenital heart disease at high risk of poor outcome. Am J Cardiol. 2014; 113(5):865-870. [PubMed: 24411285]

17. Gatzoulis MA, Till JA, Somerville J, Redington AN. Mechanoelectrical interaction in tetralogy of Fallot: QRS prolongation relates to right ventricular size and predicts malignant arrhythmias and sudden death. Circulation. 1995; 92:231-237. [PubMed: 7600655]

18. Khairy P, Fernandes SM, Mayer JE, Triedman JK, Walsh EP. Long-term survival, modes of death, and predictors of mortality in patients with Fontan surgery. Circulation. 2008; 117:85-92. [PubMed: 18071068]

19. Dimopoulos K, Diller GP, Petraco R, Koltsida E, Giannakoulas G, et al. Hyponatremia: A strong predictor of mortality in adults with congenital heart disease. European Heart Journal. 2010; 31:595-601. [PubMed: 19933692]

20. Bolger AP, Sharma R, Li W, Leenarts M, Kalra P, et al. Neurohormonal activation and the chronic heart failure syndrome in adults with congenital heart disease. Circulation. 2002; (106):92-99. [PubMed: 12093776]

21. Dimopoulos K, Diller GP, Koltsida E, Pijuan-Domenech A, Papadopoulou S, et al. Prevalence, predictors, and prognostic value of renal dysfunction in adults with congenital heart disease. Circulation. 2008; 117:2320-2328. [PubMed: 18443238]

22. Dimopoulos K, Diller GP, Ginnakoulas G, Petraco R, Chamaidi A, et al. Anemia in adults with congenital heart disease relates to adverse outcome. J Am Coll Cardiol. 2009; 54(22):2093-2100. [PubMed: 19926019] 


\section{1 moderate/complex CHD}

\section{Excluded 261 without CPX}

\section{Excluded 11 without other components of HFSS}

Figure 1. Patient Selection

After excluding patients without $\mathrm{CPX} *$ data and parameters required to calculate the $\mathrm{HFSS} \dagger$, 169 patients were included in the study.

${ }^{*} \mathrm{CPX}$, cardiopulmonary exercise test. ${ }^{\dagger}$ Heart Failure Survival Score. 


\section{Other}

$11 \%$

\section{Complex}

Cyanotic

$8 \%$
Repaired TOF

$43 \%$

\section{Fontan \\ $10 \%$}

\section{Systemic
$14 \%$}

\section{Ebstein}

\section{Anomaly}

Figure 2. Distribution of congenital heart lesions in the study cohort

The study cohort comprises of patients with moderate to complex congenital heart disease.

The majority of the patients had repaired TOF*

*Tetralogy of Fallot. 


\section{Composite}

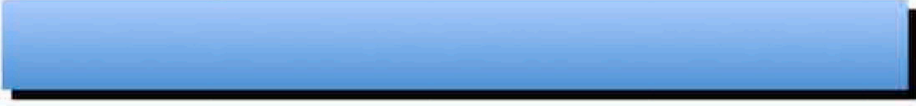

Death $\longrightarrow 5(3.0 \%)$

\begin{tabular}{l|l} 
VAD/Transplant & $0(0 \%)$
\end{tabular}

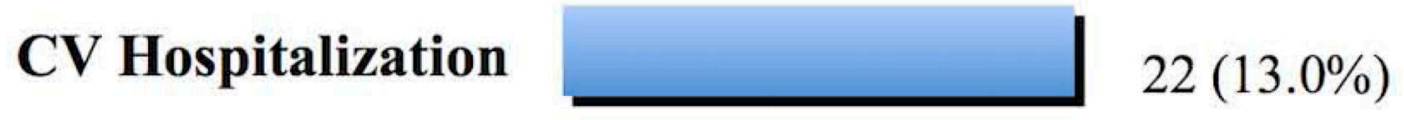

\section{Arrhythmia Req. Treatment}

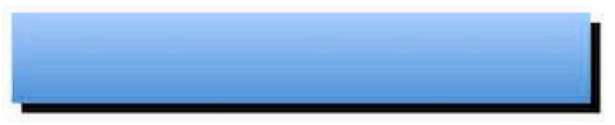

Figure 3. Outcomes in the study group

Various outcomes observed in the study cohort over a median follow-up of 2.75 years. 
Without Composite Outcome
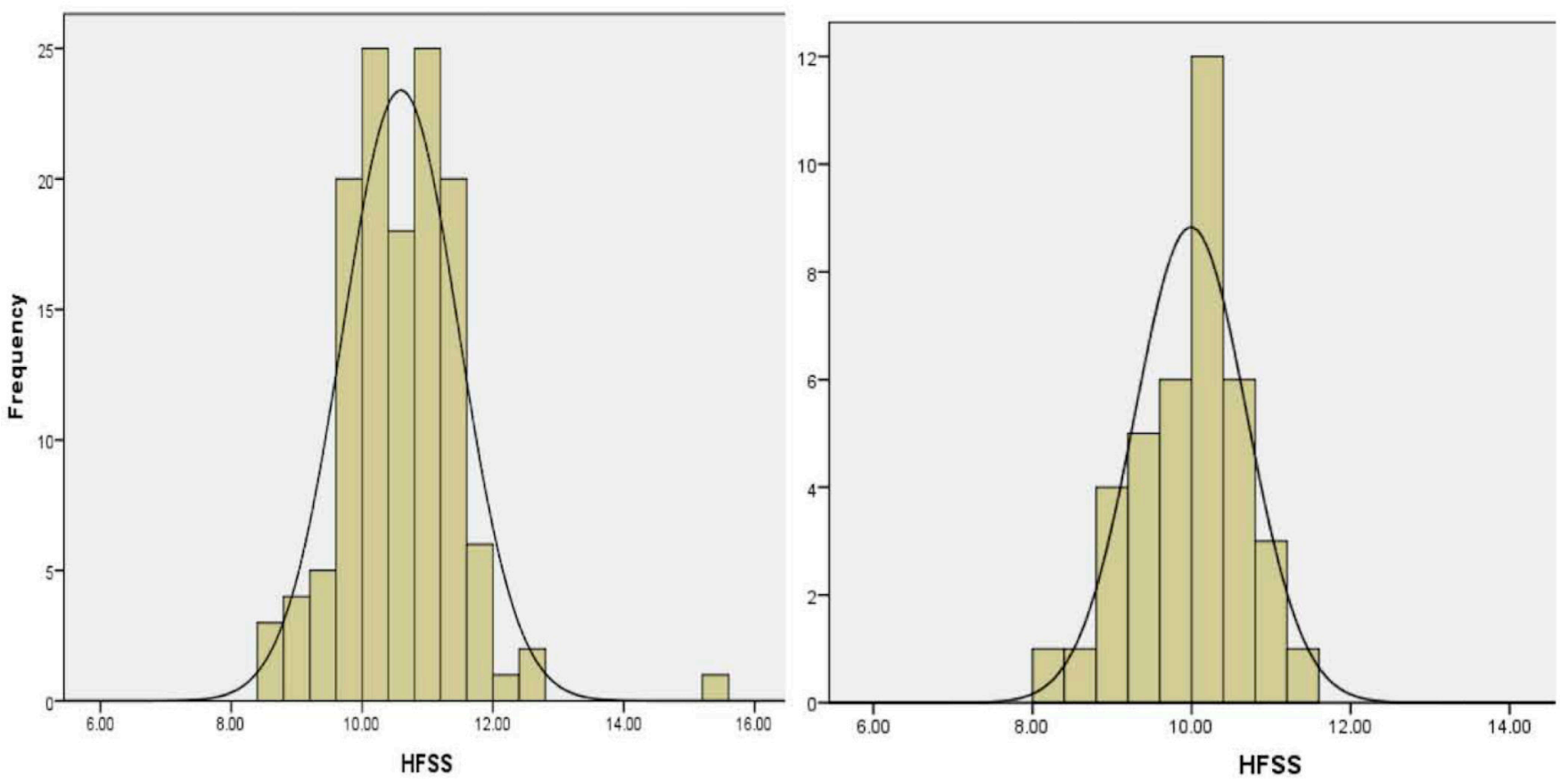

Mean $\pm \mathrm{sd}$ HFSS $=10.6 \pm 0.89 \quad \mathrm{p}<0.001 \quad$ Mean $\pm \mathrm{sd}$ HFSS $=10.0 \pm 0.70$

Figure 4. HFSS in the study group

Distribution of HFSS* in patients with and without composite outcomes.

*Heart Failure Survival Score. 


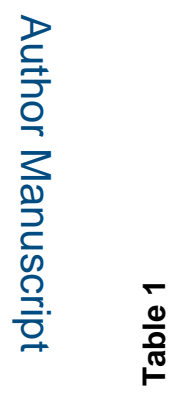

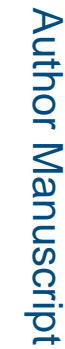

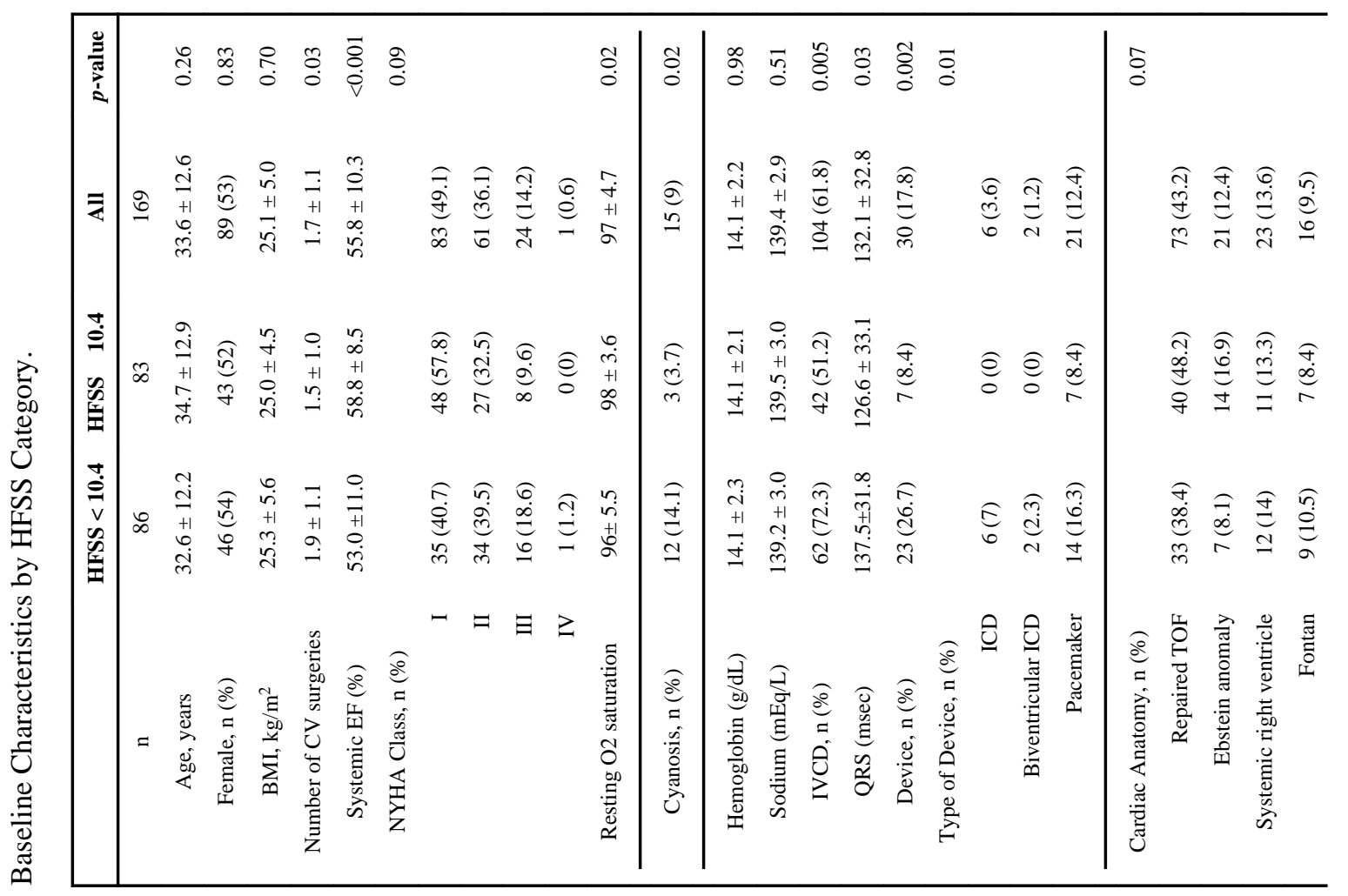

Congenit Heart Dis. Author manuscript; available in PMC 2016 September 01. 
Table 2

Selected Baseline Characteristics by Composite Event

Baseline characteristics associated with the composite events.

\begin{tabular}{ccccc}
\hline & $\begin{array}{c}\text { No Composite } \\
\text { Event }\end{array}$ & Composite Events & All & $p$-value \\
\hline $\mathrm{n}$ & 130 & 39 & 169 & \\
HFSS & $10.6 \pm 0.9$ & $10.0 \pm 0.7$ & $10.5 \pm 0.9$ & $<0.001$ \\
HR Reserve & $70.2 \pm 25.8$ & $51.9 \pm 30.5$ & $65.9 \pm 28.0$ & $<0.001$ \\
Peak VO2 & $22.6 \pm 7.3$ & $19.4 \pm 7.8$ & $21.8 \pm 7.5$ & 0.02 \\
Systemic EF & $56.8 \pm 10.0$ & $52.6 \pm 10.5$ & $55.8 \pm 10.2$ & 0.02 \\
History of CV Hospitalization & $12(9.2 \%)$ & $14(35.9 \%)$ & $26(15.4 \%)$ & $<0.001$ \\
History of Arrhythmia & $16(12.3 \%)$ & $12(30.8 \%)$ & $28(16.6 \%)$ & 0.007 \\
Use of Spironolactone & $3(2.3 \%)$ & $8(20.5 \%)$ & $11(6.5 \%)$ & $<0.001$ \\
Use of Diuretic & $18(13.8 \%)$ & $15(38.5 \%)$ & $33(19.5 \%)$ & 0.001 \\
NYHA class >2 & $12(9.2 \%)$ & $13(33.3 \%)$ & $25(14.8 \%)$ & $<0.001$ \\
Presence of Device & $17(13.1 \%)$ & $13(33.3 \%)$ & $30(17.8 \%)$ & 0.004 \\
\hline
\end{tabular}

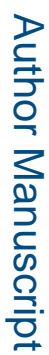

Congenit Heart Dis. Author manuscript; available in PMC 2016 September 01. 
Table 3

HFSS $^{*}$ parameters in the study group

Mean values of the HFSS components in the study cohort.

\begin{tabular}{ll}
\hline & $\mathbf{n = 1 6 9}$ \\
\hline Ischemic Cardiomyopathy & none \\
Presence of IVCD & $61.8 \%$ \\
Resting Heart Rate (bpm) & $71 \pm 13$ \\
Systemic Ventricular EF (\%) & $55.8 \pm 10.3$ \\
MeanArterial Pressure (MAP) (mm Hg) & $83 \pm 9.8$ \\
Serum Sodium (mEq/L) & $139.4 \pm 2.9$ \\
${\text { Peak } \mathrm{VO}_{2}(\mathrm{~mL} / \mathrm{kg} / \mathrm{min})}$ & $21.8 \pm 7.5$ \\
\hline
\end{tabular}

Note: Values are mean $\pm \mathrm{sd}$ or $\%$

* Heart Failure Survival Score 
Table 4

Outcomes in Patients with HFSS * $<10.4$

Odds ratios for various outcomes in patients with lower HFSS.

\begin{tabular}{lccc} 
& O.R. & $\mathbf{9 5 \%}$ CI & $p$-value \\
\hline Arrhythmia requiring treatment & 3.6 & $1.4-9.6$ & 0.007 \\
CV Hospitalization & 3.8 & $1.3-11.0$ & 0.008 \\
VAD or Transplant & $\dagger$ & & \\
Death & + & & \\
Composite & 3.7 & $1.7-8.2$ & 0.001 \\
\hline * Heart Failure Survival Score. & & \\
${ }^{\dagger}$ No VAD or transplants occurred in the study cohort. \\
${ }^{*}$ Odds ratio cannot be calculated for death as all deaths occurred in patients with HFSS $<10.4$.
\end{tabular}




\section{Table 5}

\begin{tabular}{|c|c|c|}
\hline & \multicolumn{2}{|c|}{ QRS characteristics of the study cohort } \\
\hline & QRS Characteristics & Number of patients, $\mathrm{n}(\%)$ \\
\hline & QRS >120 ms & \\
\hline & RBBB & $103(60.9)$ \\
\hline & LBBB & $1(0.01)$ \\
\hline & Non-specific IVCD & $28(16.6)$ \\
\hline & Paced rhythm & $11(6.5)$ \\
\hline \multirow{2}{*}{ 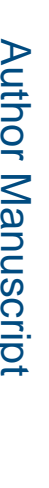 } & QRS $<120 \mathrm{~ms}$ & $26(15.4)$ \\
\hline & Total & 169 \\
\hline
\end{tabular}

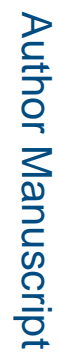

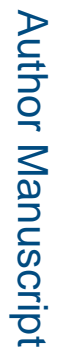

D 
Table 6

Differences in key characteristics between ACHD study cohort and patients with acquired heart failure (HF) from the HFSS study validation sample

\begin{tabular}{|l|c|c|}
\hline & Acquired HF & ACHD study cohort \\
\hline Age (y) & $52 \pm 10$ & $34 \pm 13$ \\
Female (\%) & 19 & 53 \\
Mean NYHA class & $2.8 \pm 0.7$ & $1.5 \pm 0.7$ \\
Systemic ventricular EF (\%) & $22 \pm 8$ & $55.8 \pm 10.3$ \\
Peak $\mathrm{VO}_{2}$ (mL/kg/min) & $15.9 \pm 4.3$ & $21.8 \pm 7.5$ \\
Ischemic cardiomyopathy (\%) & 47 & 0 \\
Mean blood pressures (mm Hg) & $83 \pm 12$ & $83 \pm 10$ \\
\hline
\end{tabular}

